\title{
Artificial Neural Network in Face Detection Human on Digital Image
}

\author{
Abdusamad Al-Marghilani \\ Department of Computer Science, Northern Border University, KSA
}

Received 2013-07-28, Revised 2013-08-20; Accepted 2013-08-30

\begin{abstract}
Method itself is proposed to be formed by series of filters. Each filter is an independent method of detection and allows you to cut off quickly the regions that do not contain the face's areas. For this purpose some of the different characteristics of the object are used in addition each subsequent part processes only promising areas of image which were obtained from the previous parts of the method. It has been tested by means of CMU/MIT test set. Analogy of speed and quality detection. There are two modifications to the classic use of neural networks in face detection. First the neural network only tests candidate regions for the face, thus dropping the search space. Secondly the window size is used in network scanning the input image is adaptive and depends on the size of the region of the candidate are implemented in Using Mat lab. The analysis of detection quality of a new method in comparison with the algorithm. The experimental results show that the proposed method the detection method, based on rectangular primitives, in quality. The proposed method, tested on a standard Test set, has surpassed all known methods in speed and quality of detection. Our approach without pre-treatment is not required because the normalization is enabled directly in the weights of the input network.
\end{abstract}

Keywords: Artificial Neural Network Faces Detection, Sifting Filter, Classifier, Cascade Model, Classifying Primitives

\section{INTRODUCTION}

\subsection{Neural Network}

Neural network appeared as the AI research and technology through joint efforts of engineers, physicists, mathematicians, computer scientists and neuroscientists. Although strands of research very much, there are basic core focus on pattern recognition and generation pattern.

Embedded in the general thrust on network architectures, is devoted to the structure of connections between entities and data dissemination. With regard to this scheme Links the main difference, we can do it between.

\subsection{Available Approaches to Face Detection Problem}

At the present day the problem of object detection on digital picture is very challenging one due to rapid development of photo-and video electronics.
Despite the fact that physical reality contains a lot of different objects, the development of detection algorithm for a more narrow class of objects-human faces-is of considerable interest. This is due to the increasing degree of automation of various processes and production systems. Thus, the particular application of the algorithm of human faces detection may be as follows: Automatic registration of visitors number in the supermarkets and entertainment centers; crossing control systems in various institutions, airports, subway; automated systems to prevent accidents, they monitor the face of vehicle's driver; man-machine intelligent interfaces.

Current demands in establishment of such systems impose strict limits on the speed of the algorithms executions, which shall operate in real time mode. Hence, the perspective problem to be solved is creation of fast and reliable algorithm of human faces detection.

In the last ten years, the dynamic elaborations are conducted in the field of images detection and there were 
offered a variety of detection methods: The method of principal components, methods with using of histograms, neural network, Bayesian networks, statistical methods. Some of these detecting algorithms are invariant ones with respect to the object, while others use such a priori knowledge about the object, like the shape, colours, relative position of parts.

The algorithm (Yi et al., 2010) is invariant one and possesses a high speed and high accuracy of detection. It work is as follows. There is a limited set of primitives and each set is some sort of template of picture analysis. At the stage of learning there are distinguished the primitives of such type, size and location in algorithm's search field, which distinguish in the best way the pictures containing the learning object and the pictures which do not contain it. A several thousand of primitives conjoined in classification tree are resulted the learning. In order to detect the image, the original picture is scanned by sliding window and for each position of the window the method, with use of the classification, determines whether the region is an image sought for or not. In order to detect the images of different size and orientation this approach should be applied not only to the original picture, but also to its modified copy.

The aim is to develop a new method for the detection of human faces, reflected in digital pictures, with high work rate and accuracy of detection. The idea of the Viola and Jones (2001) and Mandal (2010) algorithm was taken as a basis of the new method. All found weaknesses in the algorithm and the use of a priori knowledge about the shape and colour of the human face have allowed to identify the possible ways to improve the performance of the algorithm and to create a new method for human faces detection.

\section{DESCRIPTION OF THE NEW METHOD}

\subsection{The Method of Pixel by Pixel Filtering of Image}

The method of pixel by pixel filtering of image is the series of filtering functions of the displaying. Each pixel of the original image, depending on its intensity and the intensity of its neighbour, corresponds to a certain value. The purpose of such filtration is to separate the regions that do not contain face's areas on the basis of the information about distribution of values of the target object.

\subsection{Application of Information about Distribution of Face Skin Colour}

In many cases, the information about distribution of target object's colour may accelerate the object detection process in several times. If you look for a face in the image, the subset of RGB colour space for regions containing skin (face) fields is 6.1\% (Mandal, 2009).

After analyzing the area of colour distribution on colour pictures, an elegant formula was found. This formula allows separating effectively the areas that do not contain images of skin (face):

$$
\begin{aligned}
& (\mathrm{R}>95) \text { AND }(\mathrm{G}>40) \text { AND }(\mathrm{B}>20) \\
& \text { AND }(\mathrm{R}-\mathrm{G}>15) \text { AND }(\mathrm{R}>\mathrm{B})
\end{aligned}
$$

\subsection{Application of Information about Object's Shape}

In many cases, the information about shape of target object may also accelerate the object detection process. As an algorithm for object's shape analysis the algorithm of Canny Edge Detector (Yi et al., 2010) was chosen. The purpose of this algorithm is to find the object's boundaries on the picture. According to the intensity of the boundaries in a certain region it can be evaluated, how complex or simple the shape of the object is. After analyzing the distribution area of face's boundaries intensity on pictures there were found the threshold rates that allow effectively separating the regions that do not contain a facial image: (Intensity $>10 \%$ ) AND (Intensity $<60 \%$ ).

\section{WEAK-CLASSIFIERS ENHANCEMENT METHOD}

Strengthening of weak-classifiers is an approach to solve the problem of images detection through the integration of weak-classifiers into a single strong one. By the power of classifier in this case we mean the effectiveness (quality) of solution of detection problem. The weak-classifiers enhancement method is based on a simple premise, i.e., to combine a number of elementary (simple) features in such manner in order to get one, but more powerful. This can be done by an algorithm AdaBoost (Mittal and Kaur, 2013). As the weak classifier the anisotropic Gaussian primitive was chosen. By quality of detection it predominates the rectangular primitive of Khor (Vijayanandh and Balakrishnan, 2010), proposed by authors of Viola and Jones (2001) and Mandal (2010) algorithm.

\subsection{Anisotropic Gaussian Primitives}

Anisotropic Gaussian primitives were first described in Peotta (Mandal and Banerjee, 2012). Where they are encouraged to apply for image compression and approximation of signals. The basic function of primitives $\mathrm{cp}(\mathrm{x}, \mathrm{y})$ : R2-R2 has the form $\Phi(\mathrm{x}, \mathrm{y})=\mathrm{xe}-(\mathrm{lx}+\mathrm{y} 2)$. 


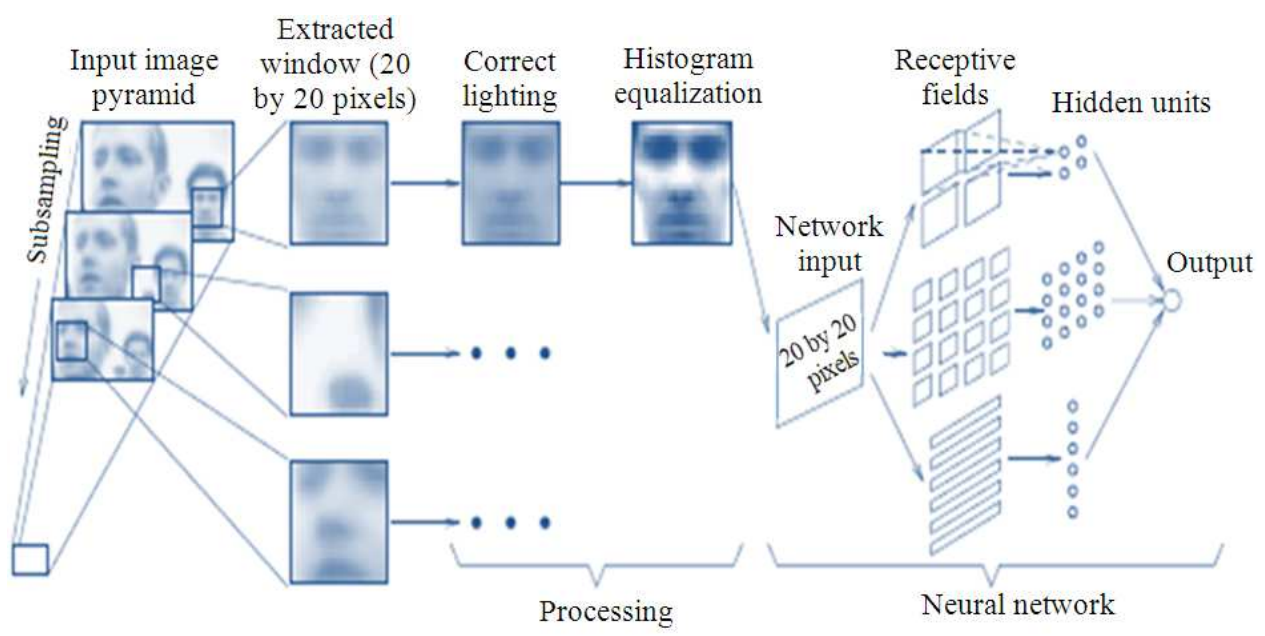

Fig. 1. Architecture for face detection are
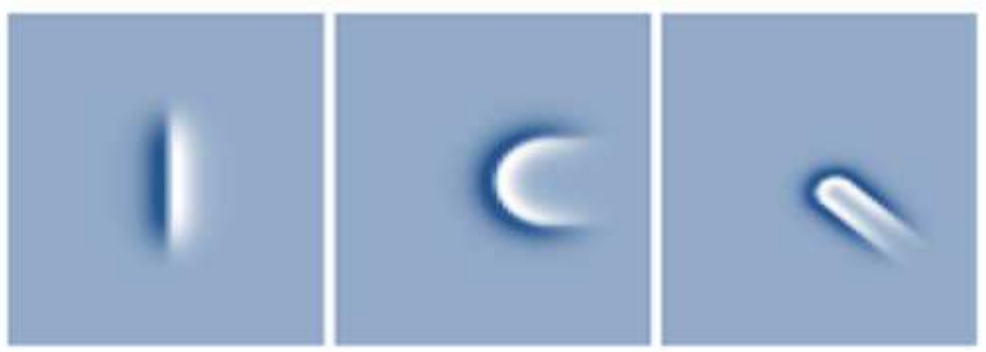

Fig. 2. Example of anisotropic Gaussian primitives

Figure 1 In addition to the basic functions, the conversion functions such as transfer, turning, bending, compression are used, which significantly expand the variety of anisotropic Gaussian primitives. Figure 2 shows examples of such primitives, obtained by different transfer parameters, rotation, bending and compression.

This class of functions has a key ability to analyze well not only the boundaries of specific areas of images, but also their gradients. Moreover, unlike the rectangular primitive of Khor (Kumar and Verma, 2010a). Its ability to analyze the boundaries is not limited to straight lines on the image.

A simple classifier based on the anisotropic Gaussian primitive is constructed as follows:

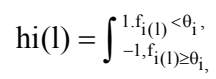

I-the signal being analyzed in the region; option $\theta$ based on Bayes rule is selected; Function $\left(\mathrm{F}_{\mathrm{i}}\right)$ is the scalar product of the region being classified and anisotropic primitive Gaussian functions:

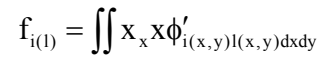

Of course, such a simple classifier cannot provide a qualitative detection of images on its own. Let us call such classifier as a weak one. In order to achieve a higher quality of detection it is necessary to combine several of them into a set of weak classifiers. Provided that each weak classifier will have its own weight. Let us call such a set as a strong classifier:

$$
\mathrm{H}_{\mathrm{M}}(\mathrm{l})=\frac{\sum_{\mathrm{m}=1} \mathrm{w}_{\mathrm{m}} \mathrm{h}_{\mathrm{m}}(\mathrm{l})}{\sum_{\mathrm{m}=1}^{\mathrm{M}} \mathrm{w}_{\mathrm{m}}}
$$

As an algorithm for constructing such a strong classifier it is proposed to use the algorithm AdaBoost (Mandal, 2009). This is an iterative algorithm, at each step of which a weak classifier is added to the strong one by weighted linear blending. The blending of classificatory is made in order to minimize the target function. In our case, we use the function of error detection in the test set. 


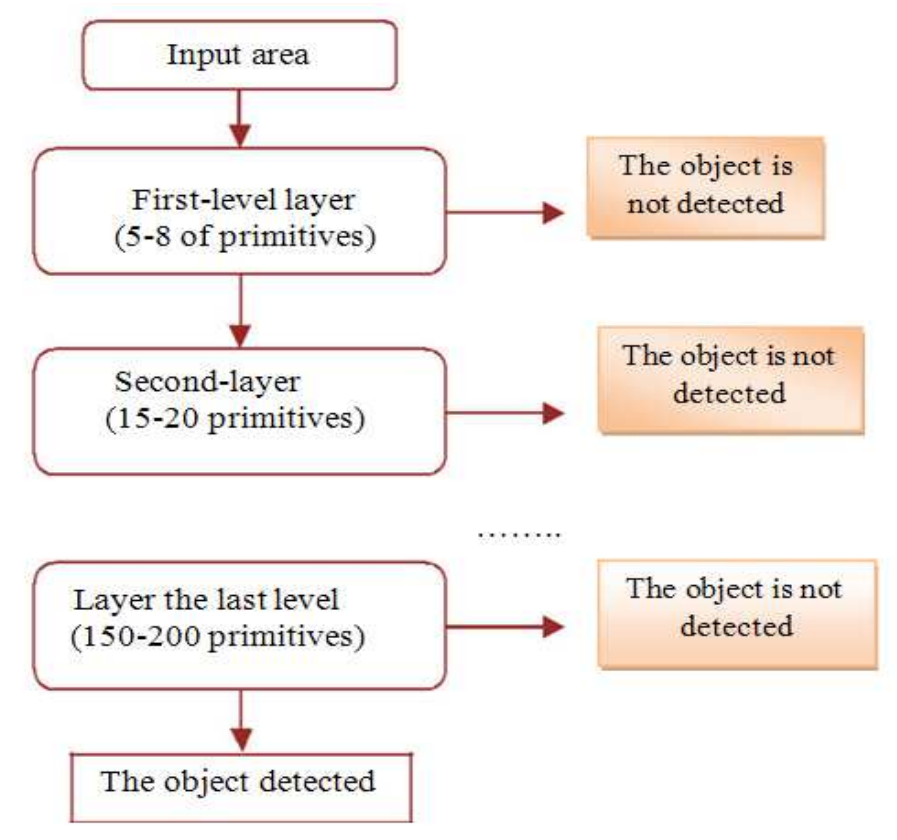

Fig. 3. Cascade model of strong classifiers

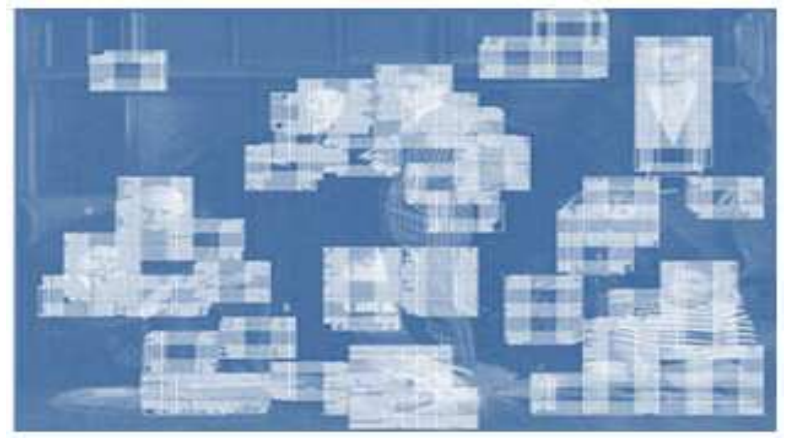

Fig. 4. Example of filtering based on a series of detectors

\subsection{Combining the Strong Classifiers into Cascade Model}

When you combine the weak anisotropic classifiers into one big strong classifier its detecting ability increases, but at that the time of decision making increases as well. In some cases, in addition to the quality of recognition, the time is a key factor. For this case it is proposed to develop a cascade model made of small strong classifiers, allowing increasing the speed of detection, without sacrificing the quality.

The cascade model of strong classifiers is a decision tree where each node of the tree is constructed to detect almost all interesting images and deflect some of the regions which are not the images. Furthermore, the tree nodes are arranged so that the closer a node is to the tree root, the smaller the amount of the anisotropic Gaussian primitives is in its composition and thus it requires less time for decision making. This type of cascade model is well suited for processing of pictures consisting of small total amount of detecting images. In this case, the method can quickly decide that the area does not contain an image and move on to the next one. The example of the cascade model is shown in Fig. 3.

\subsection{The Hybrid Method Based on a Series of Detectors}

The quality and speed of weak classifiers enhancement method directly dependent on the choice of classifying primitives. Anisotropic Gaussian primitives possess a good quality of detection, but they lose to rectangular primitives (Mursi et al., 2009) in speed the many cases, a preliminary analysis of the region can be performed by using simple primitives. In order to achieve extra performance the use of series of detectors is effective enough. In this case, the search of face with a certain pitch is performed not throughout the picture but within the area, with some margin, received from previous detector. In order to search for face in the picture it is effectively to apply a series of three detectors. The preliminary analysis is performed by 
using the primitives in window $36 \times 36$ size. Next level detector localizes the face by sequential viewing with sliding window of size $24 \times 24$ using the primitives as well. Further on the direct detection is done by anisotropic Gaussian primitives.

The result of this filtering is shown in Fig. 4.

\section{EXPERIMENTAL RESULTS}

Tests were performed on a dedicated test set CMU/MIT Test set. This set consists of 130 pictures, containing of 507 faces in total. As an opponent the algorithm Viola and Jones (2001) and Vijayanandh and Balakrishnan (2010), built on a rectangular primitives of Khor (Vijayanandh and Balakrishnan, 2010), has been chosen. This algorithm is considered to be the best universal algorithm for images detecting.

The first experiment shows the analysis of speed of face detection with use of proposed method (Table 1).

The second experiment shows the analysis of quality of face detection with use of proposed methods (Table 2).

The analysis of the experimental results showed that the error of detection has decreased with the use of additional methods.

Of detection this phenomenon can be explained by the decreasing number of false positives while the quality of faces detection as a whole remained the same.

Table 1. Analysis of speed of face detection

\begin{tabular}{lr}
\hline Method & Speed increase, times \\
\hline Only weak-classifiers & 3.8 \\
enhancement method (AdaBoost). & \\
AdaBoost + information & \\
about shape and colour of the object & 21.70 \\
AdaBoost + series of detectors & 6.50 \\
New method (hybrid one) & 28.90 \\
\hline
\end{tabular}

Table 2. Analysis of quality of face detection

\begin{tabular}{lc}
\hline Method & Erroe (\%) \\
\hline $\begin{array}{l}\text { Only weak-classifiers } \\
\text { enhancement method (AdaBoost) }\end{array}$ & 6.6 \\
$\begin{array}{l}\text { AdaBoost + information about } \\
\text { shape and colour of the object }\end{array}$ & 6.2 \\
AdaBoost + series of detectors & 6.5 \\
New method (hybrid one) & 6.1 \\
\hline
\end{tabular}

Table 3. Analysis of detection speed of a new method in comparison with the algorithm Viola and Jones (2001)

\begin{tabular}{ll}
\hline Method & Detecting speed rate $\mathrm{p} / \mathrm{s}$ \\
\hline New method (hybrid one) & 28.90 \\
Viola and Jones (2001) and & 17.44 \\
Vijayanandh and Balakrishnan (2010) & \\
\hline
\end{tabular}

The third experiment (Table 3) shows the analysis of detection speed of a new method in comparison with the algorithm Viola and Jones (2001) and Kumar et al. (2011). The proposed method predominates the detection method, based on rectangular primitives of Khor, in speed. The third experiment (Table 3) shows the analysis of detection quality of a new method in comparison with the algorithm. The experimental results show that the proposed method predominates the detection method, based on rectangular primitives of Khor, in quality.

\section{CONCLUSION}

A new method of images detection represented herein combines three ideas: the use of anisotropic Gaussian set of primitives, the combination of received sets into a cascade model for speed increase without sacrificing the quality and the use of a series of additional methods of images detection for pre-filtering of the image. The proposed method, tested on a standard test set (Kumar and Verma, 2010b). Has surpassed all known methods in speed and quality of detection.

\section{REFERENCES}

Kumar, T. and K. Verma, 2010a. A theory based on conversion of RGB image to gray image. Int. J. Comput. Appli., 7: 5-12. DOI: 10.5120/1140-1493

Kumar, T. and K. Verma, 2010b. Analysis of soft computing techniques for face detection, Ms. Thesis, Thapar University.

Kumar, T., K.V. Singh and S. Malik, 2011. Article: Artificial neural network in face detection. Int. J. Comput. Appli., 14: 5-7. DOI: 10.5120/1827-2426

Mandal, S.N. and K. Banerjee, 2012. Performance analysis for detection and location of human faces in digital image with different color spaces for different image formats. Int. J. Image, Graphics Signal Process., 4: 15-25.

Mandal, S.N., 2009. Performance analysis for detection and location of human faces in digital image with different color spaces. Proceedings of the 12th International Conference on Information Technology, (CIT' 09).

Mandal, S.N., 2010. A new technique for detection and location of human faces in digital image. Proceedings of the 4th National Conference on Computing For Nation Development, Feb. 25-26, Bharati Vidyapeeth, New Delhi, pp: 1-4. 
Mittal, H.S. and H. Kaur, 2013. Face recognition using PCA and neural network. Int. J. Emerg. Sci. Eng. 1: 71-75.

Mursi, M.F.M., M.R. Ghazy, A. Assassa and K.A. Alhumaimeedy, 2009. Automatic human face counting in digital color images. Proceedings of the 8th WSEAS International Conference on Signal Processing, Robotics and Automation, (RA' 09), ACM Press, Stevens Point, Wisconsin, USA., pp: 269-275.

Vijayanandh, R. and G. Balakrishnan, 2010. Human face detection using color spaces and region property measures. Proceedings of the 11th International Conference Control, Automation, Robotics and Vision, Dec. 7-10, IEEE Xplore Press, Singapore, pp: 16051610. DOI: 10.1109/ICARCV.2010.5707306
Viola, P. and M. Jones, 2001. Rapid object detection using a boosted cascade of simple features. Proceedings of the IEEE Computer Society Conference on Computer Vision and Pattern Recognition, (CVPR' 01), IEEE Xplore Press, pp: I511-I-518. DOI: 10.1109/CVPR.2001.990517

Yi, Y., D. Qu and F. Xu, 2010. Face detection method based on skin color segmentation and facial component localization. Proceedings of the 2nd International Asia Conference on Informatics in Control, Automation and Robotics, Mar. 6-7, IEEE Xplore Press, Wuhan, pp: 64-67. DOI: 10.1109/CAR.2010.5456778 